\title{
OUR EXPERIENCES WITH B CELL CLONALITY ANALYSIS USING MULTIPLEX PCR AMPLIFICATION IN PARAFFIN-EMBEDDED TISSUE
}

\author{
Stanek $\mathrm{L}^{1}$, Lisova $\mathbf{S}^{1}$, Tvrdik D. ${ }^{1}$ \\ ${ }^{1}$ Institute of Pathology, $1^{\text {st }}$ Faculty of Medicine, Charles University and General Faculty Hospital in Prague, Czech \\ Republic
}

\section{A b s t r a t}

The clonal determination of B-cell lymphoproliferative diseases by immunoglobulin heavy chain (IgH) rearrangement by polymerase chain reaction (PCR) is widely used. In this study we report our experiences with B cell clonality analysis using multiplex PCR amplification followed by heteroduplex analysis, which was found to be essential for the efficient resolution of monoclonal bands within polyclonal backgrounds, in formalin-fixed paraffinembedded tissue.

Key words: monoclonality, PCR, IgH, paraffin-embedded tissue, heteroduplex

\section{INTRODUCTION}

Clonality analysis of B cells using polymerase chain reaction (PCR) amplification of immunoglobulin genes has become an important tool for the diagnosis of problematical B cell lymphoproliferative diseases (1). Valuable diagnostic information is also provided by immunohistochemical techniques, especially the analysis of immunoglobulin (Ig) kappa and lambda light chains in B-cell proliferations (2). Molecular analysis of immunoglobulin gene rearrangements by Southern blotting has also proven useful in the diagnosis of B-cell neoplasms (3) althougth PCR is replacing more cumbersome hybridisation techniques.

The Ig gene contains many different variable (V), diversity (D), and joining (J) gene segments, which are rearranged during early lymphoid differentiation (4). The V-D-J rearrangement are mediated via the recombinase enzyme complex which recognizes and cutt the DNA at the recombination signal sequences, which are located downstream of the $\mathrm{V}$ gene segments, on both sides of the D gene segments, and upstream of the $\mathrm{J}$ gene segments (van Dongen 2003). The many different combinations of V, D, and $J$ gene segments resulted in combinatorial repertoire, which is estimated to be $2 \times 10^{6}$ for Ig molecules (5) that are unique in both length and sequence. Therefore, it is difficult to employ a single primer set to target all of the conserved flanking regions around V-J rearrangement and multiplex PCR reactions, which target several FR regions, which are required to identify the majority of clonal rearrangements.

The Ig heavy (IgH) chain gene has been the preferred target because most B cell tumors have rearranged IgH genes (6), (7). However, a large proportion of B cell tumors (up to 30\% depending on lymphoma type and grade) are not amenable to amplification of IgH because of the loss of target sequences or alteration as a result of somatic hypermutation (7). Therefore some authors (8) advise combined IgH and IgG gene rearrangement analysis which improved sensitivity from $66 \%$ to $85 \%$.

In this study we describe our experiences with B cell clonality analysis using multiplex PCR amplification of IgH followed by heteroduplex analysis in paraffin-embedded tissue.

Corresponding a u thor:

Libor Staněk, Institute of Pathology, Studničkova 2, 12800 Prague, Czech Republic

Tel: +420224968692; e-mail: stanek.libor@seznam.cz 


\section{MATERIAL AND METHODS}

\section{Patient and tumor samples}

This study included 25 patients surgically treated for lymphoproliferative diseases at the General Faculty Hospital (Prague, Czech Republic). Immediately after the surgical removal, the tumor specimens were frozen in liquid nitrogen and used directly for molecular analysis or they were formalin-fixed, paraffin-embedded for later use. Diagnosis was based on the standard histopathological criteria according to WHO classification (9), (10) from the histological point of view, it was mostly MCL, FL and DLBCL lyphomas with additional immunohistochemistry a panel of antibodies including CD3 1:50, CD7 1:50, CD8 1:25, CD20 1:100, CD23 1:50 (Dako) and cyclin D1, BCL6 and BCL2 (manual processing), CD4 1:20, CD5 1:50, CD15 1:40, CD30 1:80 (Roche) (robotic processing Ventana), according previous work (11), (12).

\section{Deparaffinizing slides}

The blocks of formalin-fixed, paraffin embedded tissue were cut using a microtom, and 9 paraffin slides (20 m thick) were placed directly into a sterile microfuge tube. Then $0.5 \mathrm{ml}$ of xylen was added, after which the specimens were mixed for 5 minutes and centrifuged for 5 minutes in a microfuge tube. The xylen was removed and added again to remove residual paraffin. After centrifugation, the tissue was washed twice in $0.6 \mathrm{ml}$ of $100 \%$ ethanol and centrifuged again. The tissue was dried by heating at $45^{\circ} \mathrm{C}$ for 3 minutes.

\section{DNA isolation}

DNA was extracted by using QIAamp DNA Mini Kit (Qiagen) according to the manufacturer protocol. This kit is based on the technology, which combines the selective binding properties of silica-gel-based membranes with the speed of microspin technology. After the end of the procedure, the DNA was stored at $-20^{\circ} \mathrm{C}$.

\section{PCR analysis}

PCR analysis was performed by using IdentiClone IGH gene clonality assay (InVivoScribe Technologies) which is based on the BIOMED-2 international study (5). This kit includes six master mixes. The IGH Tube A, B, and C master mixes target the framework 1, 2, and 3 within the variable region, and the joining region of immunoglobuline heavy chain locus. The IGH Tube D and $\mathrm{E}$ master mixes target the diversity and joining regions, respectively. Finally, the control size ladder master mix, targets multiple genes and generates a series of amplicons of 96, 199, 299, 399, and 608 bp to ensure the quality and quantity of the input DNA. The complete list of primer sets (originally described in van Dongen et al. 2003) including their sequences, annealing temperatures, and PCR product sizes are presented in Table I.

PCR conditions were : initial denaturation at $95^{\circ} \mathrm{C}$ for 7 minutes, followed by 35 cycles with the program of denaturation at $95^{\circ} \mathrm{C}$ for 45 seconds, annealing at $60^{\circ} \mathrm{C}$ for 45 seconds, and extension at $72{ }^{\circ} \mathrm{C}$ for 90 seconds. The reaction was accomplished with a final extension at $72^{\circ} \mathrm{C}$ for 10 minutes.

\section{Heteroduplex analysis}

For heteroduplex analysis, PCR products were denatured at $94^{\circ} \mathrm{C}$ for 5 minutes and subsequently cooled at $4^{\circ} \mathrm{C}$ for 60 minutes to induce homo and/or heteroduplexes. Briefly, the duplexes were immediately loaded on a $6 \%$ non-denaturing polyacrylamide gel in 0.5 Tris- 
Boric acid-EDTA (TBE) buffer, and run at room temperature. The visualization of products was made by SYBR Safe DNA gel stain (Invitrogen, Molecular Probes) and PCR ethidium bromid (Top-Bio, s.r.o.)

\section{FACS analysis}

A cell suspension of lymph node cells was prepared under aseptic conditions by mechanical disaggregation through tissue grinder. Samples were lysed with the hypotonic solution of $\mathrm{NH} 4 \mathrm{Cl}$ and washed in PBS containing $1 \%$ bovine serum albumin and $0.2 \%$ sodium azide (PBSA). Cell suspensions $\left(5 \times 10^{6} / \mathrm{ml}\right)$ were incubated with flurochrome-conjugated monoclonal antibodies (MoAbs) $10 \mu \mathrm{L}$ at room temperature in the dark for 15 minutes, then washed once in PBSA. After centrifugation, the cell pellet was resuspended in PBS with 1\% paraformaldehyde. All antibodies were obtained from Beckman Coulter (Fullerton, CA, USA). MoAbs used for three-colour flowcytometric analysis (FITC- fluorescein isothiocyanate, PE- phycoerythrin, PC5- phycoerythrin-cyanin 5.1): anti-CD3-PC5 (clone UCHT1), antiCD4-FITC (clone 13B8.2), anti-CD5-PE (clone BL1a), anti CD-8-PE (clone B9-11), antiCD10-FITC (clone ALB1), anti-CD11c-PE (clone BU15), anti-CD14-PE (clone RMO52), antiCD19-PC5 (clone J4.119), anti-CD20-FITC (clone B9E9), anti-CD20-PE (clone B9E9), antiCD22-PE (clone SJ10.1H11), anti-CD23-FITC (clone 9P25), anti-CD38-FITC (clone T16), anti-CD38-PE (clone LS198-4-3), anti-CD43-FITC (clone DFT1), anti-CD45-FITC (clone J.33), anti-CD71-PE (cloneYDJ1.2.2), anti-CD79a-PE (clone HM47), anti-CD79b-PE (clone CB3-1), anti-FMC-7-FITC (clone FMC7), anti-kappa-FITC (polyclonal), anti-lambda-PE (polyclonal), appropriate isotype immunoglobulin controls (Beckman Coulter). Samples were analyzed by a FACSCalibur flow cytometer (Becton Dickinson, San Jose, CA, USA) calibrated with CaliBRITE TM beads and AutoCOMP TM software (version 4.2). FACS results were obtained with CELLQuestTM software, version 3.3 (Becton Dickinson). Isotype-matched negative controls were used in all the assays to determine positive from negative cells. Expression of antigens was evaluated on the neoplastic cells by gating B lymphocytes (CD19+) on CD19 versus side scatter (SSC) dotplot. Positive expression $\geq 20 \%$ B- cells was used as cut-off. Cases were classified as monoclonal using Ig- and Ig- light-chain ratio. The ratio ( / ) is greater than 4:1 in case clone kappa. The ratio ( / ) is greater than 2:1 in case clone lambda.

\section{FACS analysis}

In each case, we initially analysed the percentage of tumor cells in each sample by FACS. In order to determine sensitivity of the method, we chose tumors with different percentages of tumor cells ( Table I). 


\section{RESULTS}

Table 1. List of primers used for PCR analysis. Specific annealing temperature $\left(\mathrm{T}_{\mathrm{a}}\right)$ of each primer and the size of expected PCR products are listed below.

\begin{tabular}{|c|c|c|c|c|c|}
\hline IGH Tube & Gene segment & Primer & Sequence (5' to $\left.3^{\prime}\right)$ & $\begin{array}{l}\text { PCR product } \\
\text { (in bp) }\end{array}$ & $\begin{array}{l}\mathrm{T}_{\mathrm{a}} \\
\left({ }^{\circ} \mathrm{C}\right)\end{array}$ \\
\hline \multirow[t]{7}{*}{ A } & VH1-FR1 & sense & GGCCTCAGTGAAGGTCTCCTGCAAG & \multirow[t]{7}{*}{$310-360$} & \multirow[t]{7}{*}{60} \\
\hline & VH2-FR1 & sense & GTCTGGTCCTACGCTGGTGAAACCC & & \\
\hline & VH3-FR1 & sense & CTGGGGGGTCCCTGAGACTCTCCTG & & \\
\hline & VH4-FR1 & sense & CTTCGGAGACCCTGTCCСТCACCTG & & \\
\hline & VH5-FR1 & sense & CGGGGAGTCTCTGAAGATCTCCTGT & & \\
\hline & VH6-FR1 & sense & TCGCAGACCCTCTCACTCACCTGTG & & \\
\hline & JH & antisense & CTTACCTGAGGAGACGGTGACC & & \\
\hline \multirow[t]{8}{*}{ B } & VH1-FR2 & sense & CTGGGTGCGACAGGCCCCTGGACAA & \multirow[t]{8}{*}{$250-295$} & \\
\hline & VH2-FR2 & sense & TGGATCCGTCAGCCCCCAGGGAAGG & & \\
\hline & VH3-FR2 & sense & GGTCCGCCAGGCTCCAGGGAA & & \\
\hline & VH4-FR2 & sense & TGGATCCGCCAGCCCCCAGGGAAGG & & \\
\hline & VH5-FR2 & sense & GGGTGCGCCAGATGCCCGGGAAAGG & & \\
\hline & VH6-FR2 & sense & TGGATCAGGCAGTCCCCATCGAGAG & & \\
\hline & VH7-FR2 & sense & TTGGGTGCGACAGGCCCCTGGACAA & & \\
\hline & $\mathrm{JH}$ & antisense & CTTACCTGAGGAGACGGTGACC & & \\
\hline \multirow[t]{8}{*}{$\mathrm{C}$} & VH1-FR3 & sense & TGGAGCTGAGCAGCCTGAGATCTGA & \multirow[t]{8}{*}{$100-170$} & \\
\hline & VH2-FR3 & sense & CAATGACCAACATGGACCCTGTGGA & & \\
\hline & VH3-FR3 & sense & TCTGCAAATGAACAGCCTGAGAGCC & & \\
\hline & VH4-FR3 & sense & GAGCTCTGTGACCGCCGCGGACACG & & \\
\hline & VH5-FR3 & sense & CAGCACCGCCTACCTGCAGTGGAGC & & \\
\hline & VH6-FR3 & sense & GTTCTCCCTGCAGCTGAACTCTGTG & & \\
\hline & VH7-FR3 & sense & CAGCACGGCATATCTGCAGATCAG & & \\
\hline & $\mathrm{JH}$ & antisense & CTTACCTGAGGAGACGGTGACC & & \\
\hline \multirow[t]{7}{*}{$\mathrm{D}$} & DH1 & sense & GGCGGAATGTGTGCAGGC & \multirow{8}{*}{$\begin{array}{l}110-290 \\
390-420\end{array}$} & \\
\hline & DH2 & sense & GCACTGGGCTCAGAGTCCTCT & & \\
\hline & DH3 & sense & GTGGCCCTGGGAATATAAAA & & \\
\hline & DH4 & sense & AGATCCCCAGGACGCAGCA & & \\
\hline & DH5 & sense & CAGGGGGACACTGTGCATGT & & \\
\hline & DH6 & sense & TGACCCCAGCAAGGGAAGG & & \\
\hline & $\mathrm{JH}$ & antisense & CTTACCTGAGGAGACGGTGACC & & \\
\hline \multirow[t]{2}{*}{$\mathrm{E}$} & DH7 & sense & CACAGGCCCCCTACCAGC & & \\
\hline & $\mathrm{JH}$ & antisense & CTTACCTGAGGAGACGGTGACC & \multirow[t]{2}{*}{$100-130$} & \\
\hline \multirow[t]{10}{*}{$\mathrm{F}$} & AF4 exon 3 & sense & GGAGCAGCATICCATCCAGC & & \\
\hline & & antisense & ACTCCATGGGCCGGACATAA & \multirow{6}{*}{$\begin{array}{c}608 \\
399 \\
299 \\
199 \\
96\end{array}$} & \\
\hline & AF4 exon 11 & sense & CCGCAGCAAGCAACGAACC & & \\
\hline & & antisense & GCTTTCCTCTGGCGGCTCC & & \\
\hline & PLZF exon 1 & sense & TGCGATGTGGTCATCATGGTG & & \\
\hline & & antisense & CGTGTCATTGTCGTCTGAGGC & & \\
\hline & RAG1 exon 2 & sense & TGTTGACTCGATCCACCCCA & & \\
\hline & & antisense & TGAGCTGCAAGTTTGGCTGAA & & \\
\hline & TBXAS1 exon 9 & sense & GCCCGACATTCTGCAAGTCC & & \\
\hline & & antisense & GGTGTTGCCGGGAAGGGTT & & \\
\hline
\end{tabular}


Table 2. Summary of clinico-pathologic and molecular findings

The abbreviations used are : MCL - mantle cell lymphoma, FL - follicular lymphoma, CLL - chronic lymphocytic leukemia, DLBCL - diffuse large B-cell lymphoma, MALT- Mucosa Associated Lymphoid Tissue, N/A - not available, FFPE - formalin-fixed paraffin-embedded

\begin{tabular}{|c|c|c|c|c|c|c|c|c|}
\hline \multirow{2}{*}{$\begin{array}{l}\text { Samp } \\
\text { le no. }\end{array}$} & \multirow{2}{*}{ Diagnosis } & \multirow{2}{*}{$\begin{array}{c}\% \\
\text { tumor } \\
\text { cells }\end{array}$} & \multirow{2}{*}{$\begin{array}{l}\text { Processing of } \\
\text { the sample }\end{array}$} & \multicolumn{5}{|c|}{ IGH gene rearrangement } \\
\hline & & & & $\begin{array}{c}\text { VH- } \\
\text { FR1/JH }\end{array}$ & $\begin{array}{c}\text { VH- } \\
\text { FR2/JH }\end{array}$ & $\begin{array}{c}\text { VH- } \\
\text { FR3/JH }\end{array}$ & $\begin{array}{l}\text { DH1- } \\
6 / J H\end{array}$ & $\mathrm{DH} 7 / \mathrm{JH}$ \\
\hline 1 & reactive lymph node & 0 & frozen tissue & - & - & - & - & - \\
\hline 2 & reactive lymph node & 0 & FFPE & - & - & - & - & - \\
\hline 3 & MCL & 86 & frozen tissue & + & + & + & - & - \\
\hline 4 & MCL & 86 & FFPE & + & + & + & - & - \\
\hline 5 & MCL & 63 & frozen tissue & + & - & + & + & - \\
\hline 6 & MCL & 63 & FFPE & + & - & + & - & - \\
\hline 7 & FL & 56 & frozen tissue & - & - & - & + & + \\
\hline 8 & MCL & 31 & frozen tissue & + & + & + & - & - \\
\hline 9 & MCL & 31 & FFPE & + & + & + & - & - \\
\hline 10 & CLL & 29 & FFPE & - & - & + & - & - \\
\hline 11 & DLBCL & 24 & FFPE & - & - & + & - & - \\
\hline 12 & DLBCL & 24 & FFPE & - & + & - & + & - \\
\hline 13 & $1 / 2 \mathrm{FL} 1 / 2$ DLBCL & 13 & FFPE & - & - & - & - & - \\
\hline 14 & $\mathrm{FL}$ & $\mathrm{N} / \mathrm{A}$ & frozen tissue & - & - & + & + & - \\
\hline 15 & FL & $\mathrm{N} / \mathrm{A}$ & FFPE & - & - & - & + & + \\
\hline 16 & CLL & 12 & FFPE & - & + & - & - & - \\
\hline 17 & DLBCL & 16 & FFPE & - & + & - & - & \\
\hline 18 & MALT & 81 & FFPE & - & - & - & + & - \\
\hline 19 & DLBCL & 53 & frozen tissue & - & + & - & - & - \\
\hline 20 & MALT & 22 & FFPE & - & + & - & - & - \\
\hline 21 & FL & 58 & FFPE & - & - & + & - & - \\
\hline 22 & FL & 47 & FFPE & - & - & + & - & - \\
\hline 23 & MCL & 28 & frozen tissue & - & + & - & - & - \\
\hline 24 & DLBCL & 32 & FFPE & - & - & - & + & - \\
\hline 25 & MALT & 46 & FFPE & - & + & - & - & \\
\hline
\end{tabular}




\section{PCR analysis of IGH rearrangements}

In order to determine the correct quality of PCR amplification, we briefly analysed the presence of the control genes in each sample. We found the presence of the 608, 399, 299, 199 and 96 base pairs PCR product corresponding to the human AF4 gene exon 3 and 11, promyelocytic leukemia zinc-finger gene (PLZF), recombination activating gene (RAG1) and tromboxane synthase gene (TBXAS1), respectively (Fig.1,2). Because smaller PCR fragments are preferentially amplified and DNA may be degraded in paraffin-embedded material, it is not unusual for the $608 \mathrm{bp}$ fragment to have a diminished signal or to be missing entirely.
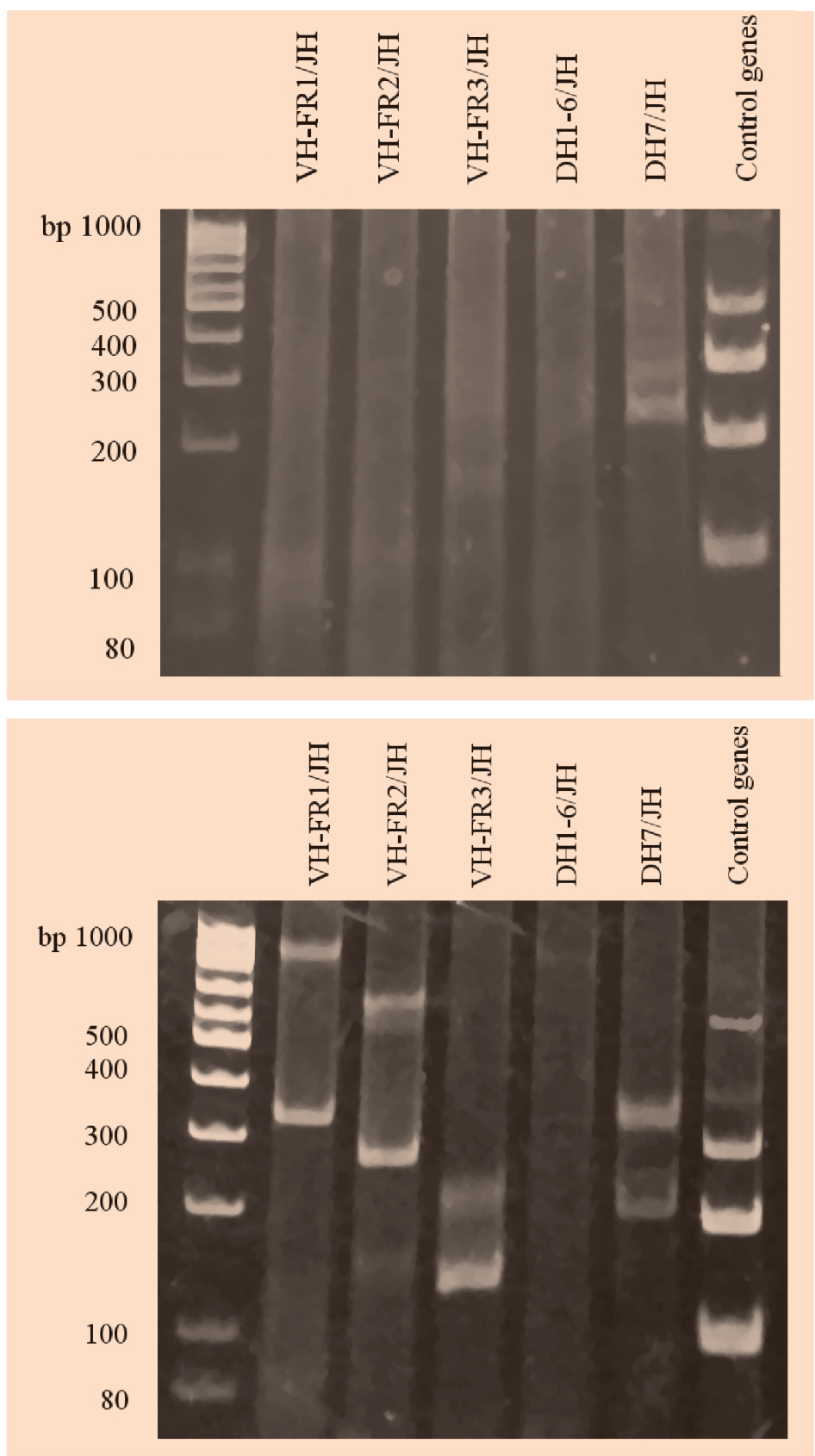

Fig. 1. PCR analysis of IGH rearrangement - polyclonal pattern DNA was extracted from sample no. 1. The analysis showed typical heteroduplex smears.
Fig. 2. PCR analysis of IGH rearrangement - monoclonal pattern DNA was extracted from sample no. 3 . The analysis showed typical homoduplex bands within the valid size range. 
We then analysed the presence of IGH rearrangements in reactive lymph nodes. We found no product or a clearly distinguishable smear without any band at the position of homoduplexes (Figure 1). We interpreted these negative results as : Negative for the detection of clonal immunoglobuline heavy chain gene rearrangements.

Finally, we analysed the presence of IGH rearrangements in the tumors (Figure 2). After heteroduplex PCR analysis with VHFR1/JH primers, a monoclonal pattern of one discrete band within the expected size range $(310-360 \mathrm{bp})$ was seen in 6 of $13(46.2 \%)$ of the cases of lymphoma using both fresh frozen and formalin-fixed paraffin-embedded tissue. The remaining 7 cases gave rise to a smear of products. Following amplification with VHFR2/JH primers one discrete band within the expected size range (250-295 bp) was seen in 5 of 13 (38.5\%) of the cases. After VHFR3/JH amplification we found one discrete band within the expected size range (100-170 bp) in 9 of 13 (69.2\%) of the cases. PCR amplifications for the $\mathrm{DH} / \mathrm{JH}$ junction showed one or two discrete bands within the expected size range (110-290 and/or 390-420, 100-130 bp) in 7 of 26 (26.9\%) of the cases.

In the remaining case, which contained only $13 \%$ tumor cells, no monoclonal bands were seen, which suggests the detection limit of this method.

In some cases, bands additional to the monoclonal homoduplex band could be seen (Figure 2). Such bands were located at a size higher than $500 \mathrm{bp}$, and so did not complicate the interpretation of the clonal bands. In addition, we did not observe any differences in the monoclonal pattern in the case of using formalin-fixed paraffin-embedded tissue when compared to the fresh frozen tissue, which indicated that the primers amplified a small amplicon (up to $400 \mathrm{bp}$ ) in order to be able to use formalin-fixed paraffin-embedded material, which often provided poor quality of extracted DNA.

In all the cases, when one or two prominent positive bands within the valid size range were seen, we interpreted these results as : Positive for the detection of clonal immunoglobuline heavy chain gene rearrangements consistent with the presence of clonal cell population.

\section{DISCUSSION}

The characterization of immunoglobulin (Ig) gene rearrangement has become an important step in the diagnosis of B-cell malignancies. Strategies for the amplification of Ig heavy chain genes have been described in a series of reports (1), (6), (7), (13), but they have not become well established, especially for the amplification of formalin-fixed paraffin-embedded material in which the DNA may be highly degraded. There are a few pitfalls in the successful amplification and interpretation of the results.

Firstly, there are false-positive results due to the background generated by the amplification of rearranged Ig genes in polyclonal cell populations. Several techniques solved this problem including e.g. direct sequencing of PCR products (14), gene scanning (5) and heteroduplex analysis (15). Among these methods, heteroduplex analysis is a simple, fast, and cheap method for the analysis of PCR products in case of a rearranged Ig gene. In heteroduplex analysis, PCR products are denatured at high temperature and subsequently renatured to induce homo- or heteroduplex formation which is essential for the efficient resolution of monoclonal bands within polyclonal backgrounds.

The second problem is false-negative results due to improper annealing of primers and/or the presence of somatic hypermutation. However, the novel BIOMED-2 multiplex PCR method for detecting B- and T-cell clonality was demonstrated to be a very reliable assay (5). In general, the analysis was performed with optimized DNA probes for the IGH locus.

Another problem which may have affected the results of PCR amplification is the input material. In general, fresh frozen tissue is considered to be the ideal sample for the extraction of DNA. However, fresh frozen material is not always available because the majority of diagnostic biopsies submitted for PCR analysis constitute formalin-fixed paraffin-embedded 
tissue. DNA extracted from formalin-fixed paraffin-embedded material is often of poor quality. The integrity of DNA extracted from paraffin-embedded samples and its amplification by PCR are affected by a number of factors such as the thickness of tissue, fixative type, fixative time, DNA extraction procedures, and the coextraction of PCR inhibitors (16),(17),(18),(19),(20). The integrity of DNA fragments extracted from paraffin-embedded samples also depends on the length of time the blocks have been stored before analysis with the best results usually obtained from blocks less than 2 years old, while blocks over 15 years old tend to yield extremely degraded fragments (21). This problem can be solved by designing of the primers which amplified the small amplicon - up to $400 \mathrm{bp}(22)$.

In conclusion, B cell clonality analysis using the BIOMED-2 multiplex PCR amplification method (van Dongen et al. 2003) followed by heteroduplex analysis is a valuable diagnostic aid for B cell lymphoproliferative diseases.

\section{REFERENCES}

1. Diss Tc, Pan L. Polymerase chain reaction in the assessment of lymphomas. Cancer Surv 1997; 30:21-44.

2. Davis Re, Warnke Ra, Dorfman Rf. Utility of molecular genetic analysis for the diagnosis of neoplasia in morphologically and immunophenotypically equivocal hematolymphoid lesions. Cancer 1991; 67:2890-2899.

3. Beishuizen A, Verhoeven Ma, Mol Ej, Breit Tm, Wolvers-Tettero Il, Van Dongen Jj. Detection of immunoglobulin heavy-chain gene rearrangements by Southern blot analysis: recommendations for optimal results. Leukemia 1993; 7:2045-2053.

4. Tonegawa S. Somatic generation of antibody diversity. Nature 1983; 302, 575-581.

5. Van Dongen Jj, Langerak Aw, Brüggemann M, Evans Pa, Hummel M, et al. Design and standardization of PCR primers and protocols for detection of clonal immunoglobulin and T-cell receptor gene recombinations in suspect lymphoproliferations: report of the BIOMED-2 Concerted Action BMH4-CT98-3936. Leukemia 2003; 17(12):2257-2317.

6. Diss Tc, Pan L, Peng H. Sources of DNA for detecting B cell monoclonality using PCR. J Clin Pathol 1994; 47:493-496.

7. Wan Jh, Trainor Kj, Brisco Mj. Monoclonality in B cell lymphoma detected in paraffin wax embedded sections using the polymerase chain reaction. J Clin Pathol 1990; 43:888-890.

8. Gong Jz, Zheng S, Chiarle R, De Wolf-Peeters C, Palestro G, et al. Detection of immunoglobulin kappa light chain rearrangements by polymerase chain reaction. An improved method for detecting clonal B-cell lymphoproliferative disorders. Am J Pathol. 1999; 155(2):355-363.

9. Weiss, S.W. (1994) WHO: Histological typing of soft tissue tumors. Berlin, Springer, 1994.

10. Elaine S. J. The 2008 WHO classification of lymphomas: implications for clinical practice and translational research Center for Cancer Research, National Cancer Institute, National Institutes of Health, Bethesda, 2008.

11. Coelho Siqueira Sa, Ferreira Alves Va, Beitler B, Otta Mm, Nascimento Saldiva Ph. Contribution of immunohistochemistry to small B-cell lymphoma classification. Appl Immunohistochem Mol Morphol. 2006;14(1):1-6.

12. Salles G, De Jong D, Xie W, Rosenwald A, Chhanabhai M, et al. Prognostic significance of immunohistochemical biomarkers in diffuse large B-cell lymphoma: a study from the Lunenburg Lymphoma Biomarker Consortium. Blood 2011; 117(26):7070-7078.

13. Trainor Kj, Brisco Mj, Wan Jh, Neoh S, Grist S, et al. Gene rearrangement in B- and T-lymphoproliferative disease detected by the polymerase chain reaction. Blood. 1991; 78(1):192-196.

14. Kneba M, Bolz I, Linke B, Hiddemann W. Analysis of rearranged T-cell receptor -chain genes by polymerase chain reaction (PCR) DNA sequencing and automated high resolution PCR fragment analysis. Blood 1995; 86:3930-3937.

15. Garcia-Sanz R, Lopez-Perez R, Langerak Aw, Gonzalez D, Chillon Mc, et al. Heteroduplex PCR analysis of rearranged immunoglobulin genes for clonality assessment in multiple myeloma. Haematologica. 1999;84(4):328335 .

16. Alaibac M, Filotico R, Giannella C, Paradiso A, Labriola A, et al. The effect of fixation type on DNA extracted from paraffin-embedded tissue for PCR studies in dermatopathology. Dermatology 1997; $195: 105-107$.

17. An Sf, Fleming Ka. Removal of inhibitor(s) of the polymerase chain reaction from formalin fixed, paraffin wax embedded tissues. J Clin Pathol 1991; 44: 924-927.

18. Longy M, Duboue B, Soubeyran P, Moynet D. Method for the purification of tissue DNA suitable for PCR after fixation with Bouin's fluid. Uses and limitations in microsatellite typing. Diagn Mol Pathol 1997; 6: $167-173$.

19. Sato V, Sugie R, Tsuchiya B, Kameya T, Natori M, et al. Comparison of the DNA extraction methods for polymerase chain reaction amplification from formalin-fixed and paraffin-embedded tissues. Diagn Mol Pathol 2001; 10: 265-271. 
20. Tbakhi A, Totos G, Pettay Jd, Myles J, Tubbs Rr. The effect of fixation on detection of B-cell clonality by polymerase chain reaction. Mod Pathol 1999; 12: 272-278.

21. Goelz Se, Hamilton Sr, Vogelstein B. Purification of DNA from formaldehyde fixed and paraffin embedded human tissue. Biochem Biophys Res Commun 1985; 130: 118-126.

22. Tvrdík, D., Svatošová, J., Dundr, P., Povýšil, C. Molecular Diagnosis of Synovial Sarcoma: Detection of SYTSSX1/2 Fusion Transcripts by RT-PCR in Paraffin-Embedded Tissue. Med. Sci. Monit. 2005; 11:1-7.

Received: February, 2, 2014

Accepted: April, 22, 2014 